\title{
Analysis of Different Braille Devices for Implementing a Cost-effective and Portable Braille System for the Visually Impaired People
}

\author{
Ruman Sarkar \\ Student, M.Tech \\ National Institute of Technology, Agartala \\ Tripura (W), India
}

\author{
Smita Das \\ Assistant Professor, CSE Department \\ National Institute of Technology, Agartala \\ Tripura (W), India
}

\begin{abstract}
There are diverse tools and equipments which are proving different facilities and features for enhancing the communication skills of the visually impaired people in a great extent. For interpretation of text by the visually impaired persons, most of the devices are designed to simulate the standard Braille display where each character is characterized by six dots (made up of small metals or plastic pins) which could be vertically moved in upper or lower directions mechanically or electronically or through any other mechanism. Also for writing the contents, same standard is followed for building any Braille writer. Analyzing different updated machinery and equipments which support the visually impaired persons for their proper communication with the others, it has been found that these tools provide only some particular facility or feature and that tools sometime may not be sufficient to provide improved support. That is why it is necessary to integrate all different possible communication methods in a single system to cover the different drawbacks in the existing systems and provide a complete solution to those visually impaired people. This paper is the result of analysis of the existing tools and technologies for the enhancement of the communication of the blind people and this paper also proposes a complete system which consists of four different techniques for the blind people to enhance their communication skills. The proposed system consists of a low cost keyboard as Braille writer, a low cost tactile refreshable Braille display (named as SPARSHA), remote communication method through SMS and an Audio output system of the text. This system may provide a complete solution to different communication problem in the visually impaired person's lives.
\end{abstract}

\section{Keywords}

Visually impaired people, Braille device, SPARSHA, Tactile display, SMS, Audio, Refreshable Braille Technology, HCI.

\section{INTRODUCTION}

From quite a long time research trends are pointing towards human computer interaction among which communication of physically challenged people plays a major role. Physically challenged people like visually impaired or deaf-blind people are facing lots of problem while communicating or interacting with other people. To provide a helping hand towards the visually impaired people's recent technological growth has been developing different skilled methods to enhance their communication procedures. For reading and writing visually impaired people always use Braille representation of different alphabets, digits, symbols etc. This international standard system of reading for the blind people has undergone for over 175 years without momentous rivalry from any other form of written communication. Braille technology has its application at automatic teller machines, elevators, hospitals, on public signs and menus, and many other, often unnoticed places. Before the initiation of Braille, the blind people read ordinary letters in relief. This method was burdensome and difficult to read anything quickly. Nevertheless, a fifteen-year-old boy named Louis Braille in 1824 in Paris, France, designed a full sixty-three-character code that used cells made of six easily producible dots. He was directly inclined by a new code shown to him by a French artillery captain Charles Barbier. The captain had developed a code what he called "night writing" for messages delivered in the dark within the army. He roughly tried to make it accessible at an instant to the blind people under the name of "sonography" and in doing so he met with Louis Braille, who developed the idea into what is today's most common reading method for the blind people ${ }^{[1]}$.

2. REFRESHABLE BRAILLE DISPLAYS In the middle of the year 1970, Refreshable Braille displays ${ }^{[2]}$ were first introduced. It was then electromechanical displays that used tiny solenoids to shift the pins. By the late 1970's, piezoelectric displays ${ }^{[3]}$ launched in the market. It was first introduced by Oleg Tretiakoff. The piezoelectric cell consists of a small ceramic substrate. The pins of that display would be activated and moved up and down when approximately 200 volts of power supply is applied. These actuators were tiny as preferred, but they were very expensive. The first popular collection of Braille display, the Versa Braille, ${ }^{[4]}$ was based on Tretiakoff's design and manufactured by Tele Sensory in the beginning of 1980's, and by the end of the 1980's Braille system was completely habituated of using piezoelectric cells [5]

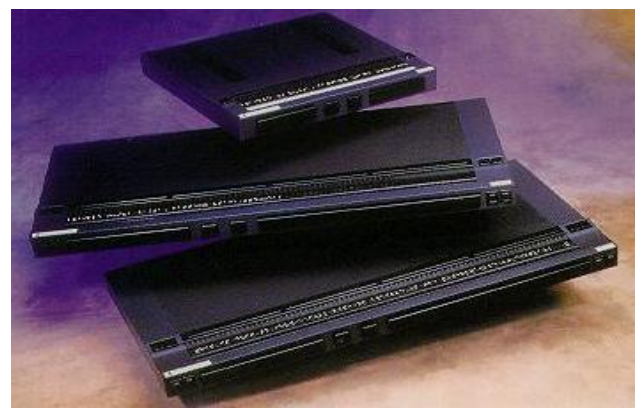

Figure 1 Refreshable Braille Displays

Though the concept of piezoelectric cells was revolutionary, still manufacturers of refreshable Braille displays have been forced all along to find a cheaper alternative to piezoelectric technology. One initiative tried by IBM was a moving Braille cell ${ }^{[6]}$, which slid along a trail and simply punched Braille 
into the finger. This was considered deplorable because the movement of the finger over the Braille is important. In another sample, dots were pushed into a moving belt of Mylar and then pressed smooth again after being read. This was attempted several times by separate companies, but was rejected because the Mylar wore out too quickly, was noisy, and felt unnatural. The pneumatic display ${ }^{[7]}$ technology a;so showed its potential for some time but was abandoned ultimately. One endeavor at cost efficiency called the Braille Mate launched by Tele Sensory eliminated all sorts of problem but it was made with one cell ${ }^{[8]}$. In the early 1990's, more experimental techniques came in the limelight, based upon mainly fluids technology and with materials which modify their integrity when a voltage is applied. In the rotating display technique ${ }^{[9]}$, the fingers stay motionless and the Braille, constantly being refreshed, moves up and down. A prototype built at NIST (National Institute of Standards and Technology) in Gaithersburg, Maryland, uses a rotating drum with the Braille cells on the perimeter of the drum. This machine uses stationary solenoids to punch specially designed pins into one of two states, up or down ${ }^{[10]}$. Among all the Braille displays that are running in the market, the most significant and competitive features are those that engage navigation through a screen of text. Some of the latest models use two-dimensional navigational control, where horizontal and vertical display positions can be altered. Other viable features include compatibility with Windows environments and speech amalgamation. Speech software, though not replacing the reading of Braille, is becoming a standard in technology for the blind. In the refreshable Braille display, however, speech synthesis is usually restricted to simple messages passing information about text attributes or graphics located at the cursor position ${ }^{[11]}$. In the preliminary part of our paper we have tried to focuses on the different existing Braille technologies for improvement of communication of the deaf-blind or blind persons/visually impaired and also we have tried to point out the different benefits, limitations and in the later part of this paper we have tried to propose a system to integrate different possible communication methods in a single system to cover the different drawbacks in the existing systems.

\section{MOTIVATION AND OBJECTIVES}

Tactile displays are the devices that stimulate the skin, particularly on fingertips, by which user can feel the shape of any object. Braille, a worldwide standard scheme for displaying text information among the deaf/blind, is by far the most successful tactile tool. A Braille cell consisting of 6 contact pins can represent a single character. In the standard Braille pattern ${ }^{[12]}$, pins are spaced $2.54 \mathrm{~mm}$ away from each other and are capable of producing vertical strokes of $0.7 \mathrm{~mm}$. Each pin produces a $170 \mathrm{mN}$ pull force at a refreshable rate of $6 \mathrm{~Hz}$. Most Braille devices commercially available are based on piezoelectric bimorph benders and relay-lever mechanisms. So one can say that, Braille displays are not usually portable. Therefore, this paper introduces a complete system in which the different subsystems of the system are portable and low cost with proper support for the visually impaired persons.

The objectives of this study are:

1. To make a complete low cost and portable system. This includes different communication tools and technologies for the visually impaired people to improve their communication skills.

2. This system includes a low cost Braille writer through which the visually impaired persons can write any text in the Braille script easily.
3. A low cost refreshable Braille display where the normal text will be displayed in Braille representation and the blind person can read easily.

4. A remote communication system through SMS service. Normal text SMS can be read in the display device in the form for Braille for the blind and the blind persons also can communicate remotely through SMS by writing in Braille which will be converted into normal text and will be sent to the non blind person. Blind to blind communication is also possible through SMS.

5. Another service is the audio output of the text. The system will read the text and it will be sent to the speaker so that the blind persons can easily understand and communicate easily. This service is only for the blind people. Those who are deafblind they cannot use this service.

\section{RECENT DEVELOPMENT}

To formulate different types of Braille reading tactile display devices special types of actuators are used such as piezoelectric ultrasonic motor for the propose of highlyportable Braille displays for stirring pin upward or downward for representing Braille or vibrators or electrochemical actuators. In present days, Body Braille system ${ }^{[13]}$ is one of the most recent inventions in Braille system technology where the actuators are accumulated on the exterior of the body and from the deformation of the skin or from the vibration of the actuators give tactile sensation which assist to stand for the Braille code for the visually impaired people. Some of the recent developments in the Braille systems are discussed below:

\subsection{Body-Braille system}

In this Body Braille system six numbers of micro vibrators are used which can normally be found in a mobile phone. They are modified to present the Braille code to be read by visually disabled people using any part of the body surface. This "Body-Braille" system can be used equally by the sensitivity of various parts of the body such as fingers, palm, ears, head, abdomen, back, arms and legs ${ }^{[14]}$. According to the research, the best parts for Braille response are the arms and back.

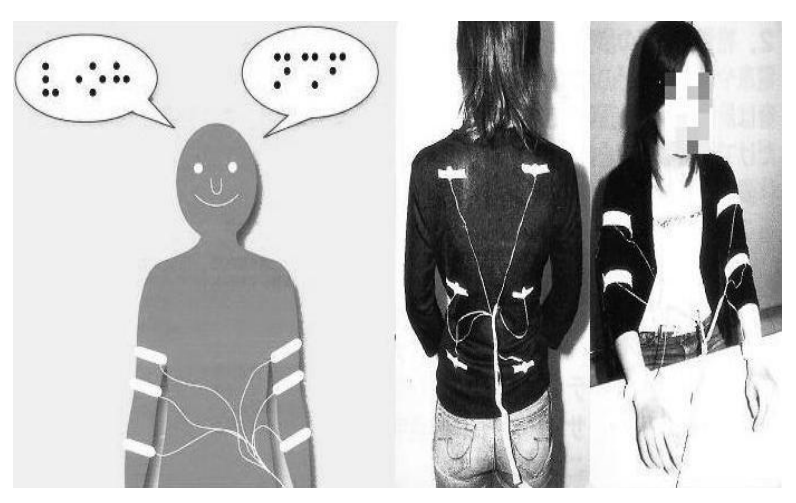

Figure 2 Mounting 6 vibrators on the different parts of the body

Figure 2 shows how the 6 vibrators are placed. One part of the Body-Braille system is called "B-brll", which consists of 8 key switches, 6 vibratos and other communication interfaces such as infrared rays, serial line. B-brll has a micro computer (PIC) inside and it controls the drive of the vibrators and handles communication interfaces. The size of "B-brll" is 
$160 \mathrm{~mm}(\mathrm{~W}), 40 \mathrm{~mm}(\mathrm{H}), 100 \mathrm{~mm}(\mathrm{D})$ and the weight is about $500 \mathrm{~g}$.

\subsection{Piezoelectric actuators}

Another development in the Braille system is the piezoelectric actuators ${ }^{[15]}$. This offers highly-portable Braille displays using a motor which consists of a shaft, a mobile element or slider, and a piezoelectric ceramic transducer and accomplishes linear movement based on the principle of inertia displacement. Unlike existing mechanisms, this motor offers a condensed, ultra-lightweight, and simple design that intends to make possible highly-portable Braille displays for the blind people.

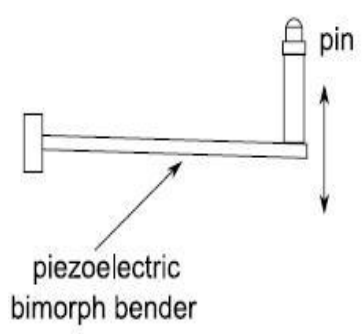

(a)

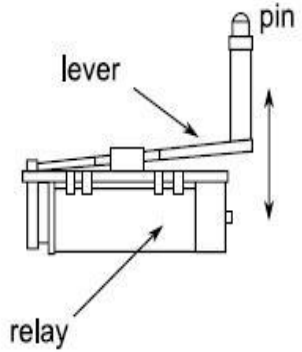

(b)
Figure 3 Examples of existing Braille mechanisms: (a) piezoelectric bimorph bender and (b) relay-lever system.

\section{PROPOSED SCHEME}

Although these devices are widely used, three drawbacks hinder the use of these devices in daily life: requirements of high-voltage supplies, poor portability because of large size of actuators and high price. Using the different services of the proposed system Deaf-blind or blind persons can communicate with the deaf-blind, blind and non-disabled persons. Likewise, non disabled persons also can communicate with the blind and deaf blind persons. This proposed system consists of four subsystems.

i) A Braille writer through which the deaf blind and the blind persons can write the Braille script.

ii) A refreshable Tactile display device named as SPARSHA to display the Braille representation of any text one character at a time.

iii) An audio output system to generate speech corresponding to any text

iv) An SMS service for remote communication.

\subsection{Working principal of the proposed model}

In case of communication between deaf-blind and nondisabled persons, the deaf-blind may type the equivalent Braille script of any text through the keyboard (Braille writer) and this Braille code will be sent to the application(control program) running on the computer and the Braille code of each character will be translated to the corresponding character (alphabet, digit, symbols etc).These translated characters together will form the actual text and will be displayed on the screen of the non-disabled person. This communication can also be done by using the SMS service. Now non-disabled person can type the English text in the application and it will be split into character and every time a character from the character array will be translated to Braille equivalent code. The control program will generate the equivalent signal and will send to the tactile display device SPARSHA through the parallel port. This SPARSHA will get the signal and then it will amplify the signal and the corresponding pins will move upward for representing the Braille code for the particular character and by sensing the pins visually impaired person can understand the character. By refreshing the device all the character will be displayed on the device.

This above communication can also be done remotely using the SMS service. Here he non-disabled person can send SMS to the deaf blind person or to the blind person and this SMS will be received by the computer attached mobile phone and automatically the application program will get the SMS text and above mention way it will be converted to the Braille code and will be displayed on the display device SPARSHA for the visually impaired person. The visually impaired person can also reply by writing his or her text in Braille representation by using the Braille writer (Braille keyboard) and this text again it will be converted into normal English text and will be sent through SMS to the non disabled person automatically.

The visually impaired person those who can hear, they can take the help of audio output system for communicating with the nondisabled persons as well as with the visually impaired persons. Here the text entered by the non-disabled persons are directly converted into speech and displayed by the audio put system i.e. through the speaker it is reached to the visually impaired person.

\subsection{SPARSHA display device architecture}

Including the existing features of the tactile display devices the following are the desired features for the proposed display:
a. Standard Cell Size
b. Fast refresh rate
c. Low cost
d. Portable
e. Small size

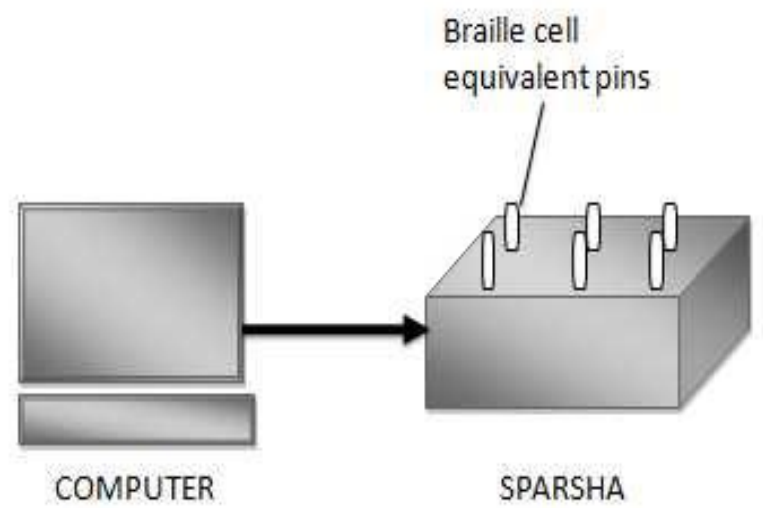

Fig. 4 SPARSHA: refreshable Braille display architecture 
The concept behind this Braille display is to represent the standard Braille code in the form of six pin which represent the six cell of Braille display technique. In this proposed model of Braille display the display device is connected to the computer system and gets the equivalent signals from the computer to display any particular character in Braille code. After getting the signal form the computer the device moves upward the particular pins for representing the particular character. For the next character the device gets refreshed and displays the next character in the similar way and so on until the text is displayed. Inside the device there are six stepper motors which work as the actuator and they helps to move the pins upward and downward. The computer has an application program to take the text in English and the application program performs different language processing, parsing, interpreting and controlling operations to meet the desire objective of the system.
The steps for the SPARSHA device architecture are depicted below:

Text processing: after getting the text from the non blind user the program split the text into array of characters and treats as individual character.

Interpreter: This individual character then assigned the standard Braille representation.

Controller: Based on the Braille representation of each character the program sends the corresponding signal to the device through the parallel port.

Inside the device: After getting the signal corresponding to any particular character the signal is first amplified to level so that the signals can drive the stepper motors.

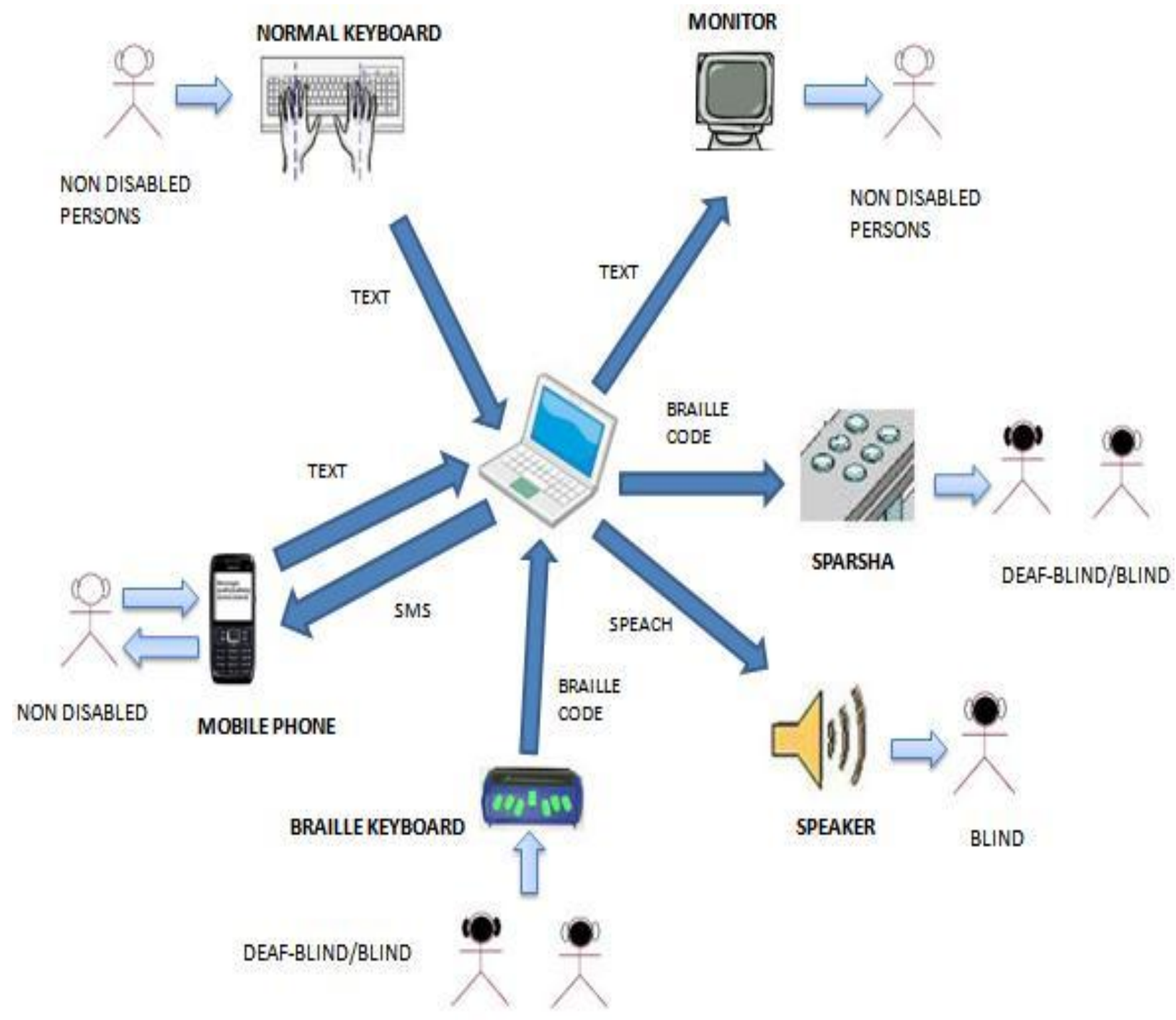

Fig 5 Block diagram of the overall system

The block diagram of the proposed system is depicted below in figure 5. It shows the non-disabled to blind, deaf/blind communication. Communication can be in the form of reading, writing or audio signal. 


\section{CONCLUSION}

Regardless of the increasing attractiveness of voice synthesis for the accessibility of textual information, Braille always resides in a preferable position as a medium for many visually impaired persons for their communication with other people. In this paper first we have studied various existing technologies and tools which can assist visually impaired people in communication with rest of the world. This learning has motivated us to think about a complete package/system by which physically disabled people can get a ready to use product for their communication. We have tried to present an idea which can provide its contributions to the development of low cost refreshable Braille display and its applications to offer an affordable portable tool for visually impaired persons to communicate with the deaf-blind, blind and non- disabled persons. Usually many of the blind people cannot afford the existing Braille reader or writer due to their high cost and importability. In our proposed scheme we have provided reading, writing, audio signal and also SMS service for remote communication. Therefore, all these service together can generate a complete package for the visually impaired people for improving their communication with rest of the world.

\section{REFERENCES}

[1] "A simplified linear refreshable Braille display", theis by Christopher W. Tullar, December, 2001.

[2] J. S. Lee and S. Lucyszyn, "A micromachined refreshable Braille cell,'J. Microelectromech. Syst., vol. 14, no. 4, pp. 673-682, August 2005.

[3] Blazie, Dean, "Refreshable Braille Now and in the Years Ahead", Braille Monitor, 43(1) (National Federation for the Blind, 2000) pp $1-6$.

[4] Stansell, Bruce, Design and Development of a Cost Efficient Braille Display, (Appalachian State University, 1997) pp. 69

[5] D. Kontarinis and R. Howe, "Tactile display of vibratory information in tele-operation and virtual environments", Presence, 4(4), pp. 387-402, 1995.
[6] www.deafblind.com

[7] The NIST Rotating-Wheel Based Refreshable Braille Display, www.nist.gov/itl/div895/isis/projects/Braille pp. $1-6$.

[8] ALVA Satellite Series, http://www.humanware.com/E/E1/E1K.html, pp. 1-2.

[9] S. Ino, S. Shimizu, T. Odagawa, M. Sato, M. Takahashi, T. Izumi and T. Ifukube, "A tactile display for presenting quality of materials by changing the temperature of skin surfaces", Proc. IEEE International Workshop on Robot and Human Communication, pp 220-224, 1993.

[10] E. Petriu and W McMath, "Tactile operator interface for semiautonomous robotic applications", Proc. of Artificial Intelligence, Robotics and Automation, pp 77-82, 1992.

[11] H. Ko, S. Kim, S. Borodinas, P. Vasiljev, C. Kang and S. Yoon, "A novel tiny ultrasonic linear motor using the radial mode of a bimorph", Sensors and Actuators A, 125, pp 477-481, 2006

[12] Velazquez, E. Preza and H. Hernandez, "Making eBooks accessible to blind Braille readers", Proc. of 2008 IEEE International Workshop on Haptic Audio Visual Environments and Games, pp 25-29, 2008.

[13] M. Goldfarb and N. Celanovic, "Modeling piezoelectric stack actuators for control of micromanipulation", IEEE Control Systems Magazine, 17, pp 69-79, 1997.

[14] Satoshi Ohtsuka, Nobuyuki Sasaki, Sadao Hasegawa, Tetsumi Harakawa, "Wearable support system for handicapped person using Body-Braille and IC-tag", RISP International Workshop on Nonlinear Circuits and Signal Processing, 2006.

[15] Hermes Hernandez et al. "Characterization of a Piezoelectric Ultrasonic Linear Motor for Braille Displays", 2009 IEEE Electronics, Robotics and Automotive Mechanics Conference 978-0-7695-3799. $3 / 09$. 